\title{
Heat and Mass Transfer Effect on MHD Flow of a Viscoelastic Fluid through a Porous Medium Bounded by an Oscillating Porous Plate in Slip Flow Regime
}

\author{
S. N. Sahoo \\ Department of Mathematics, Institute of Technical Education and Research, Siksha "O" Anusandhan University, Khandagiri, \\ Bhubaneswar, Odisha 751030, India
}

Correspondence should be addressed to S. N. Sahoo; sachi.sahoo@yahoo.com

Received 30 March 2013; Accepted 18 June 2013

Academic Editor: Jose C. Merchuk

Copyright $(2013$ S. N. Sahoo. This is an open access article distributed under the Creative Commons Attribution License, which permits unrestricted use, distribution, and reproduction in any medium, provided the original work is properly cited.

\begin{abstract}
Unsteady flow of an eclectically conducting and incompressible viscoelastic liquid of the Walter $B^{\prime}$ model with simultaneous heat and mass transfer near an oscillating porous plate in slip flow regime under the influence of a transverse magnetic field of uniform strength is presented. The governing equations of the flow field are solved by a regular perturbation method for small elastic parameter, and the expressions for the velocity, temperature, concentration, skin friction $C_{f}$, the heat flux in terms of the Nusselt number $N_{u}$, and the rate of mass transfer in terms of the Sherwood number $S_{h}$ are obtained. The effects of the important flow parameters on the dynamics are discussed. Findings of the study reveal that the rarefaction parameter accelerates the fluid particles in the flow domain. Elastic parameter contributes to sudden fall of the velocity near the plate. Magnetic force contributes to greater skin friction as the time elapses. Destructive reaction reduces, whereas generative reaction enhances the concentration distribution.
\end{abstract}

\section{Introduction}

Free convective flow in presence of heat source has been a subject of interest of many researchers because of its possible application to geophysical sciences, astrophysical sciences, and in cosmical studies. Such flows arise either due to unsteady motion of the boundary or the boundary temperature. The study of fluctuating flow is important in the paper industry and many other technological fields. Therefore, many researchers have paid their attention towards the fluctuating flow of viscous incompressible fluid past an infinite plate. Singh et al. [1] have analyzed the heat and mass transfer in MHD flow of a viscous fluids past a vertical plate under oscillatory suction velocity. Sharma and Singh [2] have reported the unsteady MHD-free convective flow and heat transfer along a vertical porous plate with variable suction and internal heat generation. The problem of slip flow regime is very important in this era of modern science, technology, and vast ranging industrialization. In many practical applications, the particle adjacent to a solid surface no longer takes the velocity of the surface. The particle at the surface has a finite tangential velocity; it slips along the surface. The flow regime is called the slip flow regime, and its effect cannot be neglected. The fluid slippage phenomenon at the solid boundaries appear in many applications such as microchannels or nanochannels and in application where a thin film of light oils is attached to the moving plates or when the surface is coated with special coating such as thick monolayer of hydrophobic octadecyltrichlosilane, that is, lubrication of mechanical device where a thin film of lubricant is attached to the surface slipping over one another or when the surfaces are coated with special coating to minimize the friction between them. Singh and Gupta [3] have discussed the MHD-free convective flow of a viscous fluid through a porous medium bounded by an oscillating porous plate in slip flow regime with mass transfer. Khandelwal and Jain [4] have analyzed the unsteady MHD flow of a stratified fluid through porous medium over a moving plate in slip flow regime. Das et al. [5] have studied the magnetohydrodynamic unsteady flow of a viscous stratified fluid through a porous medium past a porous flat moving plate in the slip flow regime with 
heat source. The study of heat and mass transfer problems with chemical reaction is of great practical importance to engineers and scientists because of their almost universal occurrence in many branches of science and engineering. A few representative fields of interest in which combined heat and mass transfer along with chemical reaction play an important role are chemical process industries such as food processing and polymer production. Mahapatra et al. [6] have studied effects of chemical reaction on free convection flow through a porous medium bounded by a vertical surface. Muthucumaraswamy [7] has studied effects of chemical reaction on a moving isothermal vertical surface with suction. Al-Odat and Al-Azab [8] have studied influence of chemical reaction on a transient $\mathrm{MHD}$-free convection flow over a moving vertical plate.

Viscoelastic fluid flow through porous media has attracted the attention of scientists and engineers because of its importance notably in the flow of the oil through porous rocks, the extraction of energy from geothermal region, and the filtration of solids from liquids and drug permeation through human skin. The flow through porous media also occurs in the ground water hydrology, irrigation and drainage problems, absorption and filtration processes in the ground water hydrology, irrigation and drainage problems, absorption and filtration processes in chemical engineering, and soil erosion and tile drainage. Chaudhary and Jain [9] have investigated the effects of the Hall current and radiation on MHD mixed convection flow of a viscoelastic fluid past and infinite vertical plate. Sahoo et al. [10] have discussed the unsteady two-dimensional MHD flow and heat transfer of an elastic-viscous liquid past an infinite hot vertical porous surface bounded by porous medium with source/sink. Kumar and Chand [11] have studied the effect of slip conditions and the Hall current on unsteady MHD flow of a viscoelastic fluid past an infinite vertical porous plate through porous medium.

The objective of the present study is to consider the viscoelastic fluid past a vertical plate in the slip flow regime packed with uniform porous matrix in the presence of a transverse magnetic field. In this paper, we consider the problem of Singh and Gupta [3] in viscoelastic fluid of Walter's $B^{\prime}$ model taking into account the effect of heat source parameter and chemical reaction parameter.

\section{Formulation and Solution of the Problem}

The physical configuration consists of an unsteady flow of an electrically conducting and incompressible viscoelastic liquid of Walter's $B^{\prime}$ model with simultaneous heat and mass transfer near an oscillating infinite porous plate in slip flow regime with heat source and chemical reaction under the influence of a transverse magnetic field of uniform strength. The $y$-axis is taken along the plate in vertical direction, and $x$-axis is perpendicular to it. A uniform magnetic field of strength $B_{0}$ is applied in the direction of $y$-axis. Let $u$ and $v$ be the velocity components in $x$ - and $y$-directions, respectively. As the plate is of infinite length, all the variables in the problem are functions of $y$ and $t$. Initially, the plate and fluid are at rest, and then the plate is set to an oscillatory motion. The Reynolds number is assumed to be very small, and the induced magnetic field due to the flow is neglected with respect to the applied magnetic field. The pressure $p$ in the fluid is assumed to be constant. If $v_{0}$ represents the suction or injection velocity at the plate, the equation of continuity is

$$
\frac{\partial v}{\partial y}=0
$$

Under the condition $y=0, v=-v_{0}$ everywhere. We have

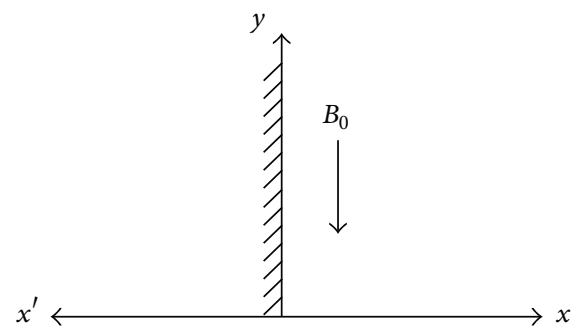

Flow Diagram

Now, the governing boundary layer equations of the flow field are

$$
\begin{gathered}
\frac{\partial u}{\partial t}-v_{0} \frac{\partial u}{\partial y}=v \frac{\partial^{2} u}{\partial y^{2}}-\sigma \frac{B_{0}^{2} u}{\rho}-\frac{v u}{K}+g \beta\left(T-T_{\infty}\right) \\
+g \hat{\beta}\left(C-C_{\infty}\right)-\frac{K_{0}}{\rho}\left(\frac{\partial^{3} u}{\partial t \partial y}+v_{0} \frac{\partial^{3} u}{\partial y^{3}}\right), \\
\frac{\partial T}{\partial t}-v_{0} \frac{\partial T}{\partial y}=\alpha \frac{\partial^{2} T}{\partial y^{2}}-S\left(T-T_{\infty}\right) \\
\frac{\partial C}{\partial t}-v_{0} \frac{\partial C}{\partial y}=D \frac{\partial^{2} C}{\partial y^{2}}-K_{l}\left(C-C_{\infty}\right) .
\end{gathered}
$$

The first order velocity slip boundary conditions of the problem when the plate executes linear harmonic oscillations in its own plane are given by

$$
\begin{gathered}
y=0: u=U_{0} e^{\text {int }}+L_{1} \frac{\partial u}{\partial y}, \quad T=T_{\infty}, \quad C=C_{\infty}, \\
y \longrightarrow \infty: u \longrightarrow 0, \quad T \longrightarrow T_{\infty}, \quad C \longrightarrow C_{\infty},
\end{gathered}
$$

where $L_{1}=\left(2-m_{1}\right)\left(L / m_{1}\right), L=\mu(\pi / 2 p \rho)^{1 / 2}$ is the mean free path, and $m_{1}$ is Maxwell's reflection coefficient. 
On introducing the following nondimensional quantities:

$$
\begin{gathered}
y^{*}=\frac{U_{0}}{v} y, \quad u^{*}=\frac{u}{U_{0}}, \quad t^{*}=\frac{U_{0}^{2}}{v} t, \\
\theta^{*}=\frac{T-T_{\infty}}{T_{w}-T_{\infty}}, \quad \phi^{*}=\frac{C-C_{\infty}}{C_{w}-C_{\infty}}, \\
v_{0}^{*}=\frac{v_{0}}{U_{0}}, \quad n^{*}=\frac{v}{U_{0}^{2}} n, \\
K_{p}=\frac{K U_{0}^{2}}{v}, \quad R=\frac{L_{1} U_{0}}{v}, \quad S^{*}=\frac{v S}{U_{0}^{2}}, \\
\frac{v K_{l}}{U_{0}^{2}}, \quad G_{r}=\frac{g \beta v\left(T_{w}-T_{\infty}\right)}{U_{0}^{3}}, \\
\left.G_{m}=\frac{g B_{0}^{2} v}{\rho U_{0}^{2}}, \quad C_{w}-C_{\infty}\right) \\
U_{0}^{3}
\end{gathered}
$$

in (2) and dropping the asterisks, we have

$$
\begin{gathered}
\frac{\partial u}{\partial t}-v_{0} \frac{\partial u}{\partial y}=\frac{\partial^{2} u}{\partial y^{2}}-R_{c}\left(\frac{\partial^{3} u}{\partial t \partial y^{3}}+v_{0} \frac{\partial^{3} u}{\partial y^{3}}\right) \\
-\left(M^{2}+\frac{1}{K_{p}}\right) u+G_{r} \theta+G_{m} \phi \\
\operatorname{Pr}\left(\frac{\partial \theta}{\partial t}-v_{0} \frac{\partial \theta}{\partial y}\right)=\frac{\partial^{2} \theta}{\partial y^{2}}-\operatorname{Pr} S \theta \\
S_{c}\left(\frac{\partial \phi}{\partial t}-v_{0} \frac{\partial \phi}{\partial y}\right)=\frac{\partial^{2} \phi}{\partial y^{2}}-K_{c} S_{c} \phi
\end{gathered}
$$

with boundary conditions

$$
\begin{aligned}
& y=0: u=e^{\text {int }}+R \frac{\partial u}{\partial y}, \quad \theta=1, \quad \phi=1, \\
& y \longrightarrow \infty: u \longrightarrow 0, \quad \theta \longrightarrow 0, \quad \phi \longrightarrow 0 .
\end{aligned}
$$

Equation (5) is of third order, and two boundary conditions are available. Due to inadequate boundary condition, a perturbation method has been applied with $R_{c}<1$ as the perturbation parameter. This assumption is quite consistent as the model under consideration is valid only for slightly elastic fluid.

Consider the following:

$$
\begin{aligned}
& u=u_{0}+R_{c} u_{1}+O\left(R_{c}\right)^{2}, \\
& \theta=\theta_{0}+R_{c} \theta_{1}+O\left(R_{c}\right)^{2}, \\
& \phi=\phi_{0}+R_{c} \phi_{1}+O\left(R_{c}\right)^{2} .
\end{aligned}
$$

Substituting (9) in (5)-(7) and equating like powers of $R_{c}$, we get the following.
Zeroth order equations:

$$
\begin{gathered}
\frac{\partial u_{0}}{\partial t}-v_{0} \frac{\partial u_{0}}{\partial y}=\frac{\partial^{2} u_{0}}{\partial y^{2}}-\left(M^{2}+\frac{1}{K_{p}}\right) u_{0}+G_{r} \theta_{0}+G_{m} \phi_{0} \\
\operatorname{Pr}\left(\frac{\partial \theta_{0}}{\partial t}-v_{0} \frac{\partial \theta_{0}}{\partial y}\right)=\frac{\partial^{2} \theta_{0}}{\partial y^{2}}-\operatorname{Pr} S \theta_{0} \\
S_{c}\left(\frac{\partial \phi_{0}}{\partial t}-v_{0} \frac{\partial \phi_{0}}{\partial y}\right)=\frac{\partial^{2} \phi_{0}}{\partial y^{2}}-K_{c} S_{c} \phi_{0}
\end{gathered}
$$

first order equations:

$$
\begin{gathered}
\frac{\partial u_{1}}{\partial t}-v_{0} \frac{\partial u_{1}}{\partial y}=\frac{\partial^{2} u_{1}}{\partial y^{2}}-\left(M^{2}+\frac{1}{K_{p}}\right) u_{1} \\
+G_{r} \theta_{1}+G_{m} \phi_{1}-v_{0} \frac{\partial^{3} u_{1}}{\partial y^{3}}-\frac{\partial^{3} u_{0}}{\partial t \partial y^{2}} \\
\operatorname{Pr}\left(\frac{\partial \theta_{1}}{\partial t}-v_{0} \frac{\partial \theta_{1}}{\partial y}\right)=\frac{\partial^{2} \theta_{1}}{\partial y^{2}}-\operatorname{Pr} S \theta_{1} \\
S_{c}\left(\frac{\partial \phi_{1}}{\partial t}-v_{0} \frac{\partial \phi_{1}}{\partial y}\right)=\frac{\partial^{2} \phi_{1}}{\partial y^{2}}-K_{c} S_{c} \phi_{1}
\end{gathered}
$$

The corresponding boundary conditions are

$$
\begin{array}{r}
y=0: u_{0}=e^{\mathrm{int}}+R \frac{\partial u_{0}}{\partial y}, \quad u_{1}=0, \quad \theta_{0}=1, \\
\theta_{1}=1, \quad \phi_{0}=1, \quad \phi_{1}=1, \\
y \longrightarrow 0: u_{0} \longrightarrow 0, \quad u_{1} \longrightarrow 0, \quad \theta_{0} \longrightarrow 0, \\
\theta_{1} \longrightarrow 0, \quad \phi_{0} \longrightarrow 0, \quad \phi_{1} \longrightarrow 0 .
\end{array}
$$

In order to reduce the system of partial differential equations (10)-(11) to a system of ordinary differential equations, we further introduce

$$
\begin{aligned}
& u_{0}(y, t)=u_{00}(y)+u_{01}(y) e^{\text {int }}, \\
& u_{1}(y, t)=u_{10}(y)+u_{11}(y) e^{\text {int }}, \\
& \theta_{0}(y, t)=\theta_{00}(y)+\theta_{01}(y) e^{\text {int }}, \\
& \theta_{1}(y, t)=\theta_{10}(y)+\theta_{11}(y) e^{\text {int }}, \\
& \phi_{0}(y, t)=\phi_{00}(y)+\phi_{01}(y) e^{\text {int }}, \\
& \phi_{1}(y, t)=\phi_{10}(y)+\phi_{11}(y) e^{\text {int }} .
\end{aligned}
$$


Substituting (13) into (10)-(11) and equating the harmonic and nonharmonic terms, we obtain

$$
\begin{aligned}
& u_{00}^{\prime \prime}+v_{0} u_{00}^{\prime}-\left(M^{2}+\frac{1}{K_{p}}\right) u_{00}=-G_{r} \theta_{00}-G_{m} \phi_{00} \\
& u_{01}^{\prime \prime}+v_{0} u_{01}^{\prime}-\left(M^{2}+\frac{1}{K_{p}}+i n\right) u_{01}=-G_{r} \theta_{01}-G_{m} \phi_{01} \text {, } \\
& u_{10}^{\prime \prime}+v_{0} u_{10}^{\prime}-\left(M^{2}+\frac{1}{K_{p}}\right) u_{10}=-G_{r} \theta_{10}-G_{m} \phi_{10}+v_{0} u_{00}^{\prime \prime \prime} \text {, } \\
& u_{11}^{\prime \prime}+v_{0} u_{11}^{\prime}-\left(M^{2}+\frac{1}{K_{p}}+i n\right) u_{11} \\
& =-G_{r} \theta_{11}-G_{m} \phi_{11}+v_{0} u_{01}^{\prime \prime \prime}+i n u_{01}^{\prime \prime} \text {, } \\
& \theta_{00}^{\prime \prime}+v_{0} \operatorname{Pr} \theta_{00}^{\prime}-\operatorname{Pr} S \theta_{00}=0, \\
& \theta_{01}^{\prime \prime}+v_{0} \operatorname{Pr} \theta_{01}^{\prime}-(i n \operatorname{Pr}+\operatorname{Pr} S) \theta_{01}=0 \\
& \theta_{10}^{\prime \prime}+v_{0} \operatorname{Pr} \theta_{10}^{\prime}-\operatorname{Pr} S \theta_{10}=0, \\
& \theta_{11}^{\prime \prime}+v_{0} \operatorname{Pr} \theta_{11}^{\prime}-(\text { in } \operatorname{Pr}+\operatorname{Pr} S) \theta_{11}=0 \\
& \phi_{00}^{\prime \prime}+v_{0} S_{c} \phi_{00}^{\prime}-K_{c} S_{c} \phi_{00}=0, \\
& \phi_{01}^{\prime \prime}+v_{0} S_{c} \phi_{01}^{\prime}-\left(i n S_{c}+K_{c} S_{c}\right) \phi_{01}=0 \\
& \phi_{10}^{\prime \prime}+v_{0} S_{c} \phi_{10}^{\prime}-K_{c} S_{c} \phi_{10}=0, \\
& \phi_{11}^{\prime \prime}+v_{0} S_{c} \phi_{11}^{\prime}-\left(i n S_{c}+K_{c} S_{c}\right) \phi_{11}=0,
\end{aligned}
$$

with boundary conditions

$$
\begin{gathered}
y=0: u_{00}=R \frac{\partial u_{00}}{\partial y}, \quad u_{01}=1+R \frac{\partial u_{01}}{\partial y}, \\
u_{10}=0, \quad u_{11}=0, \quad \theta_{00}=1, \\
\theta_{01}=0, \quad \theta_{10}=1, \quad \theta_{11}=0, \\
\phi_{00}=1, \quad \phi_{01}=0, \quad \phi_{10}=0, \quad \phi_{11}=0, \\
y \longrightarrow 0: u_{00} \longrightarrow 0, \quad u_{01} \longrightarrow 0, \quad u_{10} \longrightarrow 0, \\
u_{11} \longrightarrow 0, \quad \theta_{00} \longrightarrow 0, \quad \theta_{01} \longrightarrow 0, \\
\theta_{10} \longrightarrow 0, \quad \theta_{11} \longrightarrow 0, \quad \phi_{00} \longrightarrow 0, \\
\phi_{01} \longrightarrow 0, \quad \phi_{10} \longrightarrow 0, \quad \phi_{11} \longrightarrow 0 .
\end{gathered}
$$

\section{Results and Discussion}

The problem of unsteady flow of an electrically conducting and incompressible viscoelastic liquid of Walter's $B^{\prime}$ model with heat and mass transfer near an oscillating infinite porous 
plate in slip flow regime with heat source and chemical reaction parameter under the influence of a transverse magnetic field of uniform strength has been considered. The effects of the flow parameters such as the Prandtl number Pr, porosity parameter $K_{p}$, magnetic parameter $M$, elastic parameter $R_{c}$, heat source parameter $S$, chemical reaction parameter $K_{c}$, thermal Grashof number $G_{r}$, the mass Grashof number $G_{m}$, the Scmidt number $S_{c}$, suction parameter $v_{0}$, and rarefaction parameter $R$ on the velocity field have been studied analytically and presented with the help of Figures 1-4. The effects of the flow parameters on the temperature field and concentration distribution have been presented in Figures 5 and 6, respectively. Further, the effects of the flow parameters on the skin friction, heat flux, and rate of mass transfer have been discussed with the help of Tables 1-3. For numerical computation, the values of $G_{r}$ are taken positive. This indicates that the study has been carried out under the influence of the cooling of the plate. Also, we have taken $n t=\pi / 2$. The interesting aspect of the problem is to study the combined effect of the flow parameters with that of the first order velocity slip boundary condition when the plate executes linear harmonic oscillation in its own plane.

Figure 1 shows the effect of the Prandtl number $(\operatorname{Pr})$, permeability parameter $\left(K_{p}\right)$ and magnetic parameter $(M)$ on velocity profile. For this figure, we have taken that $S=1, G_{r}$ $=5, G_{m}=5, S_{c}=0.24, R_{c}=0.5, K_{c}=2, R=0.2$, and $v_{0}=$ 2. It is observed that the increase in the Prandtl number as well as permeability parameter decreases the velocity of the flow field, whereas increase in magnetic parameter increases it. Since Prandtl number is the ratio of kinematic viscosity to thermal diffusivity, so as Pr increases, the kinematic viscosity of the fluid dominates the thermal diffusivity of the fluid which leads to decreasing the velocity of the flow field. The application of transverse magnetic field sets up the Lorentz force, which enhances the fluid velocity.

Figure 2 shows the effect of elastic parameter $\left(R_{c}\right)$, heat source parameter $(S)$, and chemical reaction $\left(K_{c}\right)$ parameter on velocity frofile. For this figure, we have taken that $\mathrm{Pr}=$ $0.71, K_{p}=1, M=2, G_{r}=5, G_{m}$, $=5 S_{c}=0.24, R=0.2$, and $v_{0}$ $=2$. It is observed that the velocity of the flow field decreases due to the presence of elastic parameter, chemical reaction parameter, and heat source parameter. For $R_{c}=S=K_{c}=0$, the present work agrees with the work of Singh and Gupta [3].

Figure 3 depicts the effect of the thermal Grashof number $\left(G_{r}\right)$, the mass Grashof number $\left(G_{m}\right)$, and the Schmidt number $\left(S_{c}\right)$, on velocity frofile. For this figure, we have taken that $\operatorname{Pr}=0.71, S=1, K_{p}=1, M=2, R_{c}=0.5, K_{c}=2, R=0.2$, and $v_{0}=2$. It is observed that for the heavier species, that is, with increasing $S_{c}$, the velocity decreases. The velocity of the flow field decreases due to the increase in the thermal Grashof number. Moreover, buoyancy effect $\left(G_{m}\right)$ due to mass transfer enhances the velocity.

Figure 4 depicts the effect of suction parameter $\left(v_{0}\right)$ and rarefaction parameter $(R)$ on velocity frofile. For this figure, we have taken that $\operatorname{Pr}=0.71, S=1, K_{p}=1, M=2, R_{c}=0.5$, $K_{c}=2, G_{r}=5, G_{m}=5$, and $S_{c}=0.24$. It is observed that the velocity of the flow field decreases due the presence of suction

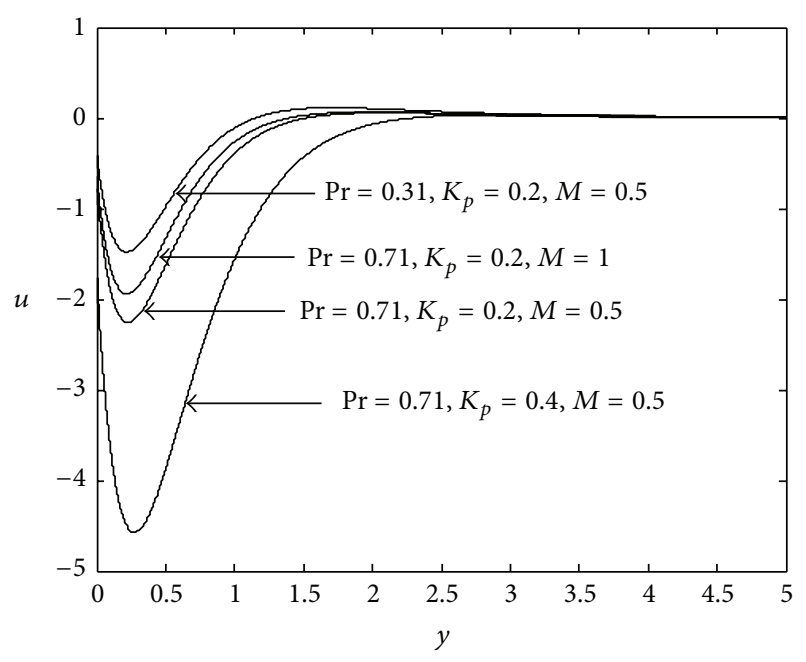

Figure 1: Effect of $\operatorname{Pr}, K_{p}$ and $M$ on velocity profile.

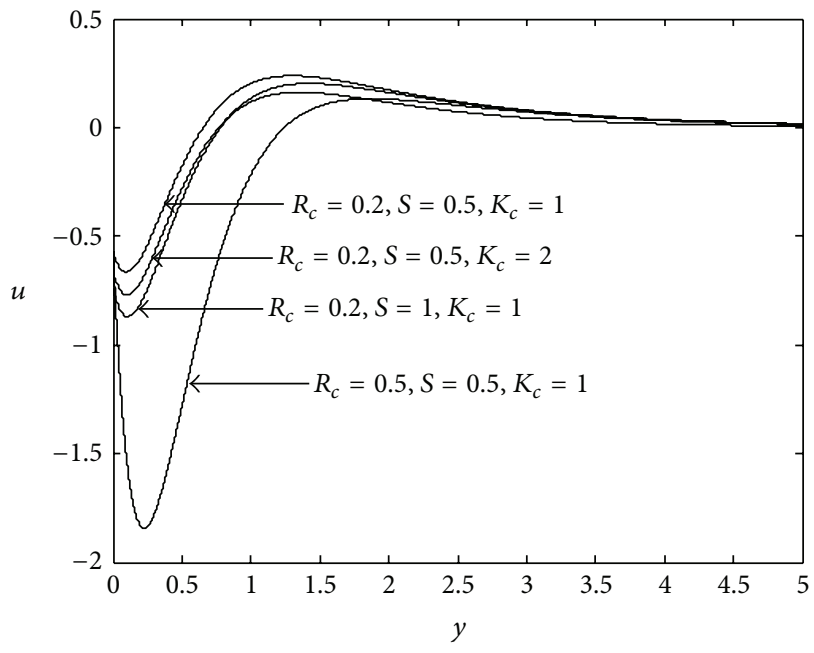

FIGURE 2: Effect of $R_{c}, S$, and $K_{c}$ on velocity profile.

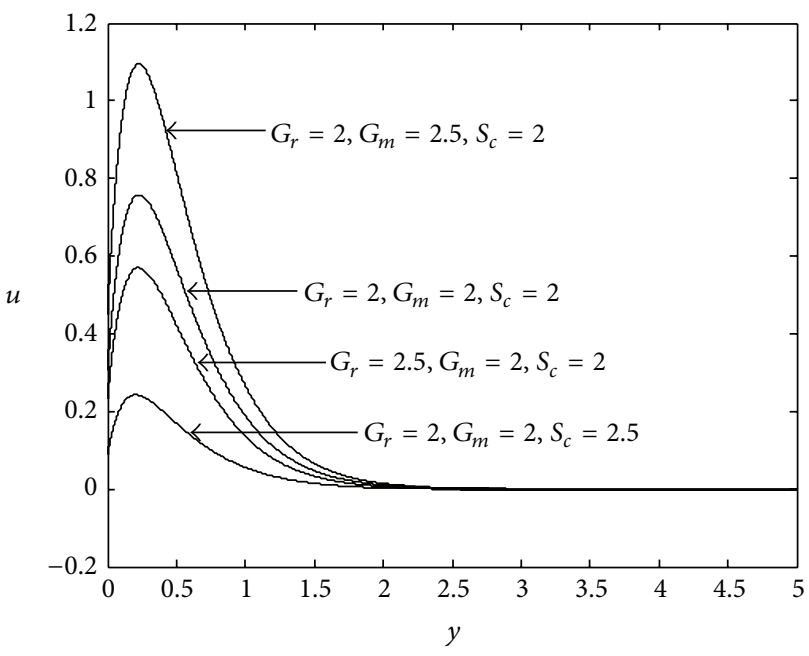

FIGURE 3: Effect of $G_{r}, G_{m}$, and $S_{c}$ on velocity profile. 


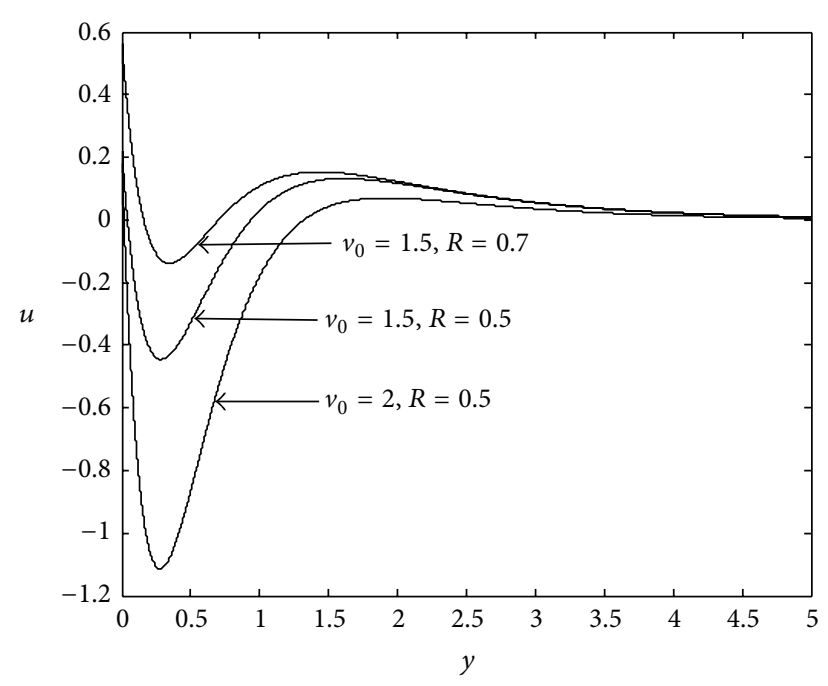

FIGURE 4: Effect of $v_{0}$ and $R$ on velocity profile.

parameter, but the reverse effect is observed due the presence of the rarefaction parameter.

Figure 5 shows the effect of the Prandtl number, heat source parameter, and suction parameter on the temperature of the flow field. It is observed that the temperature of the flow field diminishes as the Prandtl number increases. This is consistent with the fact that the thermal boundary layer thickness decreases with increasing Prandtl number. Presence of heat source reduces the temperature of the flow field. This may happen due the elastic property of the fluid. It is observed that temperature of the flow field diminishes as the suction parameter increases.

Figure 6 depicts the effect of the Schmidt number, chemical reaction parameter, and suction parameter on concentration distribution. The concentration distribution decreases at all points of the flow field with the increase in the Schmidt number. This shows that the heavier diffusing species have a greater retarding effect on the concentration distribution of the flow field. It is observed that a destructive reaction $\left(K_{c}>0\right)$ reduces the concentration distribution, whereas a generative reaction $\left(K_{c}=0\right)$ enhances it. Also, it is observed that presence of suction parameter diminishes the concentration distribution.

The skin friction is an important phenomenon which characterizes the frictional drag at the solid surface. From Table 1, it is observed that the skin friction decreases with the increase in all the forcing forces, but it is interesting to note that the skin friction increases with the increase in magnetic parameter.

From Table 2, it is to note that all the entries are positive. It is seen that the Prandtl number (Pr), heat source $(S)$ and suction parameter $\left(v_{0}\right)$ increase the rate of heat transfer at the surface of the plate.

From Table 3 it is to note that all the entries are positive. It is observed that Schmidt number $\left(S_{c}\right)$, chemical reaction parameter $\left(K_{c}\right)$, and suction parameter $\left(v_{0}\right)$ increase the rate of mass transfer at the surface of the plate.

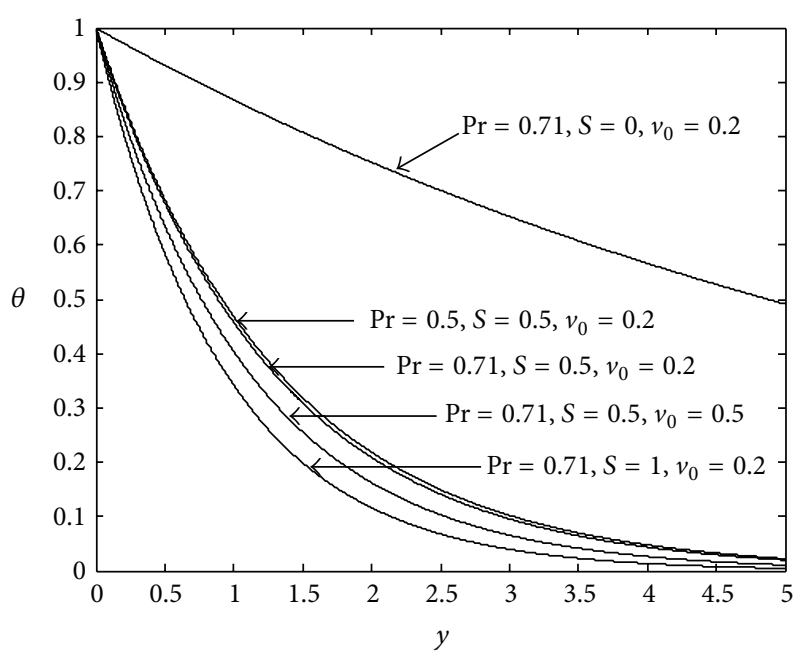

Figure 5: Effect of $\operatorname{Pr}, S$, and $v_{0}$ on temperature profile.

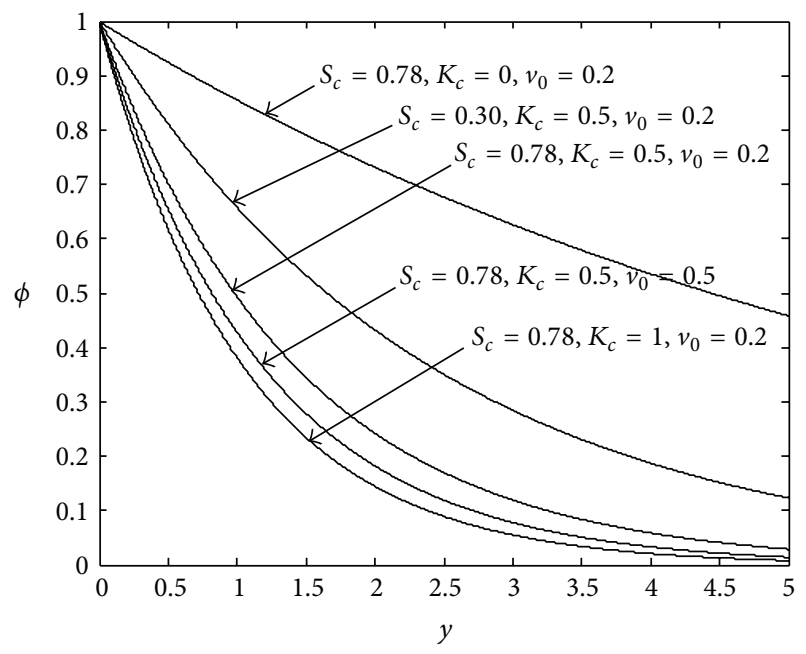

FigURE 6: Effect of $S_{c}, K_{c}$, and $v_{0}$ on concentration profile.

\section{Conclusion}

A theoretical study of unsteady MHD incompressible viscoelastic liquid of Walter's $B^{\prime}$ model with heat and mass transfer near an oscillating infinite porous plate in slip flow regime under the influence of a transverse magnetic field of uniform strength is considered. Some of the important findings of the problem are given in the following.

(i) Presence of the Prandtl number decreases the velocity of the flow field, whereas presence magnetic field increases it.

(ii) The velocity of the flow field decreases suddenly near the plate due to the presence of elastic parameter.

(iii) The velocity of the flow field decreases due to the increase in the thermal Grashof number. 
TABLE 1: Skin friction $\left(C_{f}\right)$.

\begin{tabular}{lccccccccccc}
\hline $\operatorname{Pr}$ & $K_{p}$ & $M$ & $R_{c}$ & $S$ & $K_{c}$ & $G_{r}$ & $G_{m}$ & $S_{c}$ & $R$ & $v_{0}$ & $C_{f}$ \\
\hline 0.30 & 1 & 2 & 0.5 & 1 & 2 & 5 & 5 & 0.24 & 0.2 & 2 & 18.3921 \\
0.50 & 1 & 2 & 0.5 & 1 & 2 & 5 & 5 & 0.24 & 0.2 & 2 & 10.8793 \\
0.50 & 1.5 & 2 & 0.5 & 1 & 2 & 5 & 5 & 0.24 & 0.2 & 2 & 7.1877 \\
0.50 & 1 & 2.5 & 0.5 & 1 & 2 & 5 & 5 & 0.24 & 0.2 & 2 & 30.4390 \\
0.50 & 1 & 2 & 0.2 & 1 & 2 & 5 & 5 & 0.24 & 0.2 & 2 & 13.0904 \\
0.50 & 1 & 2 & 0.5 & 0.5 & 2 & 5 & 5 & 0.24 & 0.2 & 2 & 17.9390 \\
0.50 & 1 & 2 & 0.5 & 1 & 1.5 & 5 & 5 & 0.24 & 0.2 & 2 & 11.6350 \\
0.50 & 1 & 2 & 0.5 & 1 & 2 & 4.5 & 5 & 0.24 & 0.2 & 2 & 13.1659 \\
0.50 & 1 & 2 & 0.5 & 1 & 2 & 5 & 4.5 & 0.24 & 0.2 & 2 & 11.8927 \\
0.50 & 1 & 2 & 0.5 & 1 & 2 & 5 & 5 & 0.30 & 0.2 & 2 & 9.1301 \\
0.50 & 1 & 2 & 0.5 & 1 & 2 & 5 & 5 & 0.24 & 0.5 & 2 & 2.75040 \\
0.50 & 1 & 2 & 0.5 & 1 & 2 & 5 & 5 & 0.24 & 0.2 & 1.5 & 14.0484 \\
\hline
\end{tabular}

TABle 2: The Nusselt number $\left(N_{u}\right)$.

\begin{tabular}{lccc}
\hline $\operatorname{Pr}$ & $S$ & $v_{0}$ & $N_{u}$ \\
\hline 0.50 & 0.5 & 0.2 & 0.75887 \\
0.71 & 0.5 & 0.2 & 0.78166 \\
0.71 & 1.0 & 0.2 & 1.07351 \\
0.71 & 0.5 & 0.5 & 0.90654 \\
\hline
\end{tabular}

TABle 3: The Sherwood number $\left(S_{h}\right)$.

\begin{tabular}{llcc}
\hline$S_{c}$ & $K_{c}$ & $v_{0}$ & $S_{h}$ \\
\hline 0.30 & 0.5 & 0.2 & 0.41845 \\
0.78 & 0.5 & 0.2 & 0.70735 \\
0.78 & 1.0 & 0.2 & 0.96461 \\
0.78 & 0.5 & 0.5 & 0.84923 \\
\hline
\end{tabular}

(iv) Thermal boundary layer thickness decreases with increasing the Prandtl number.

(v) Heavier diffusing species have a greater retarding effect on the concentration distribution.

\section{Appendix}

Consider the following:

$$
\begin{gathered}
a_{1}=\frac{1}{2}\left[v_{0} \operatorname{Pr}+\sqrt{\left(v_{0} \operatorname{Pr}\right)^{2}+4 S \operatorname{Pr}}\right], \\
a_{2}=\frac{1}{2}\left[v_{0} S_{c}+\sqrt{\left(v_{0} S_{c}\right)^{2}+4 K_{c} S_{c}}\right], \\
a_{3}=\frac{1}{2}\left[v_{0}^{2}+\sqrt{v_{0}^{2}+4 Q}\right], \quad Q=M^{2}+\frac{1}{K_{p}}, \\
a_{4}=\frac{1}{2}\left[v_{0}^{2}+\sqrt{v_{0}^{2}+4(Q+i n)}\right],
\end{gathered}
$$

$$
\begin{aligned}
& A_{1}=\frac{-G_{r}}{a_{1}^{2}-a_{1} v_{0}-Q}, \quad A_{2}=\frac{-G_{r}}{a_{2}^{2}-a_{2} v_{0}-Q}, \\
& A_{3}=\frac{-1}{1+a_{3} R}\left[\left(a_{1} R+1\right) A_{1}+\left(a_{2} R+1\right) A_{2}\right] \text {, } \\
& A_{4}=\frac{1}{1+R a_{4}}, \quad A_{5}=\frac{a_{1}^{3} A_{1} v_{0}}{a_{1}^{2}-a_{1} v_{0}-Q}, \\
& A_{6}=\frac{a_{2}^{3} A_{2} v_{0}}{a_{2}^{2}-a_{2} v_{0}-Q}, \quad A_{7}=A_{5}+A_{6}-\frac{v_{0} a_{3}^{3} A_{3} y}{v_{0}-2 a_{3}}, \\
& A_{8}=\frac{\left(-v_{0} a_{4}^{3} A_{4}+i n a_{4}^{2} A_{4}\right) y}{v_{0}-2 a_{4}}
\end{aligned}
$$

\section{Nomenclature}

$x, y$ : Coordinate axes

$u, v$ : Velocity components in $x$ - and $y$-directions

$t$ : Time variable

$\mu$ : Dynamic viscosity

$v$ : Kinematic viscosity

$\alpha$ : Thermal conductivity

$p$ : Pressure

g: Acceleration due to gravity

$\beta$ : Coefficient of volume expansion

$\widehat{\beta}$ : Coefficient of volume expansion with concentration

$U_{0}$ : Reference velocity

$T$ : Dimensional temperature

$\theta$ : Nondimensional temperature

$C$ : Dimensional concentration

$\phi$ : Nondimensional concentration

Pr: The Prandtl number

$G_{r}$ : The thermal Grashof number

$G_{m}$ : The mass Grashof number

$S_{c}$ : The Schmidt number

$M$ : Magnetic parameter

$S: \quad$ Heat source/sink parameter

$K_{l}$ : Dimensional chemical reaction parameter

$K_{c}$ : Nondimensional chemical reaction parameter

$T_{w}$ : Temperature at the wall

$T_{\infty}$ : Temperature far away from the wall

$C_{w}$ : Concentration at the wall

$C_{\infty}$ : Concentration far away from the wall

$\sigma: \quad$ Electric conductivity

$B_{0}$ : Uniform magnetic field

$v_{0}$ : Suction/injection velocity

$D$ : Mass diffusion

$\rho:$ Density

$K$ : Dimensional porosity parameter

$K_{p}$ : Nondimensional porosity parameter

$K_{0}$ : Dimensional elastic parameter

$R_{c}$ : Nondimensional elastic parameter

$n$ : Frequency of oscillation. 


\section{Acknowledgment}

The author wish to express his special thanks to Professor G. C. Dash, S “O” A University, Bhubaneswar, Odisha, India for his valuable suggesion and constant encouragement to complete the work.

\section{References}

[1] A. K. Singh, A. K. Singh, and N. P. Singh, "Heat and mass transfer in MHD flow of a viscous fluid past a vertical plate under oscillatory suction velocity," Indian Journal of Pure and Applied Mathematics, vol. 34, no. 3, pp. 429-442, 2003.

[2] P. R. Sharma and G. Singh, "Unsteady MHD free convective flow and and heat transfer along a vertical porous plate with variable suction and internal heat generation," International Journal of Applied Mathematics and Mechanics, vol. 4, no. 5, pp. $1-8,2008$.

[3] P. Singh and C. B. Gupta, "MHD free convective flow of viscous fluid through a porous medium bounded by an oscillaing porous plate in slip flow regime with mass transfer," Indian Journal of Theoretical Physics, vol. 53, no. 2, pp. 111-120, 2005.

[4] A. K. Khandelwal and N .C. Jain, "Unsteady MHD flow of stratified fluid through porous medium over a moving plate in slip flow regime," Indian Journal of Theoretical Physics, vol. 53, no. 1, pp. 25-35, 2005.

[5] S. S. Das, L. K. Mishra, and P. K. Mishra, "Effect of heat source on MHD free convection flow past an oscillating porous plate in the slip flow regime," International Journal of Energy and Environment, vol. 2, no. 5, pp. 945-952, 2011.

[6] N. Mahapatra, G. C. Dash, S. Panda, and M. Acharya, "Effects of chemical reaction on free convection flow through a porous medium bounded by a vertical surface," Journal of Engineering Physics and Thermophysics, vol. 83, no. 1, pp. 130-140, 2010.

[7] R. Muthucumaraswamy, "Effects of a chemical reaction on a moving isothermal vertical surface with suction," Acta Mechanica, vol. 155, no. 1-2, pp. 65-70, 2002.

[8] M. Q. Al-Odat and T. A. Al-Azab, "Influence of chemical reaction on a transient MHD free convection flow over a moving vertical plate," The Journal of Engineering Research, vol. 12, no. 3, pp. 15-21, 2007.

[9] R. C. Chaudhary and P. Jain, "Hall effect on MHD mixed convection flow of a visco-elastic fluid past and infinite vertical plate with mass transfer and radiation," Theoretical and Applied Mechanics, vol. 33, no. 4, pp. 281-309, 2006.

[10] S. N. Sahoo, J. P. Panda, and G. C. Dash, "Unsteady two dimensional MHD flow and heat transfer of an elastic-viscous liquid past an infinite hot vertical porous surface bounded by porous medium with source/sink," A.M.S.E. France, vol. 80, no. 2, pp. 26-42, 2011.

[11] R. Kumar and K. Chand, "Effect of slip conditions and Hall current on unsteady MHD flow of a viscoelastic fluid past an infinite vertical porous plate through porous medium," International Journal of Engineering Science and Technology, vol. 3, no. 4, pp. 3124-3133, 2011. 

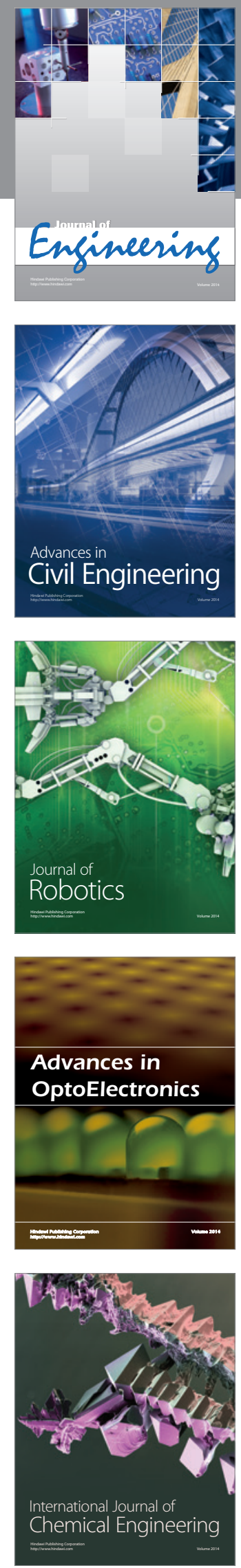

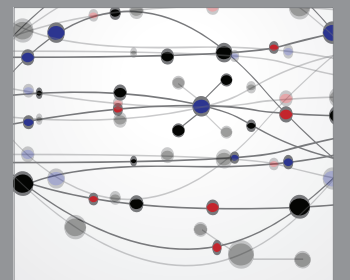

The Scientific World Journal
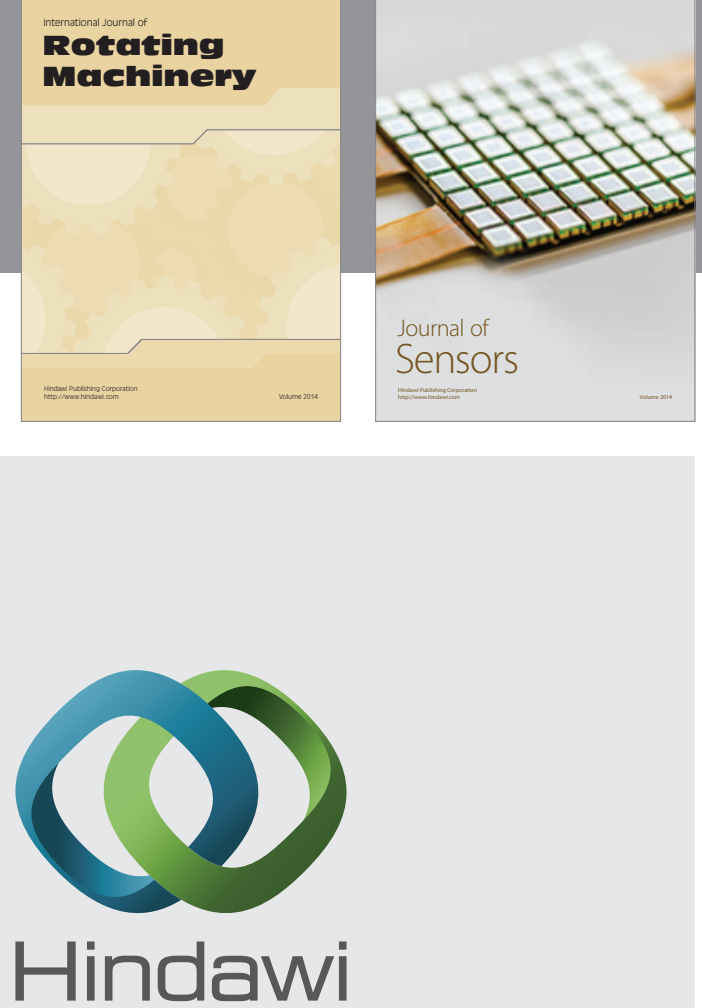

Submit your manuscripts at http://www.hindawi.com
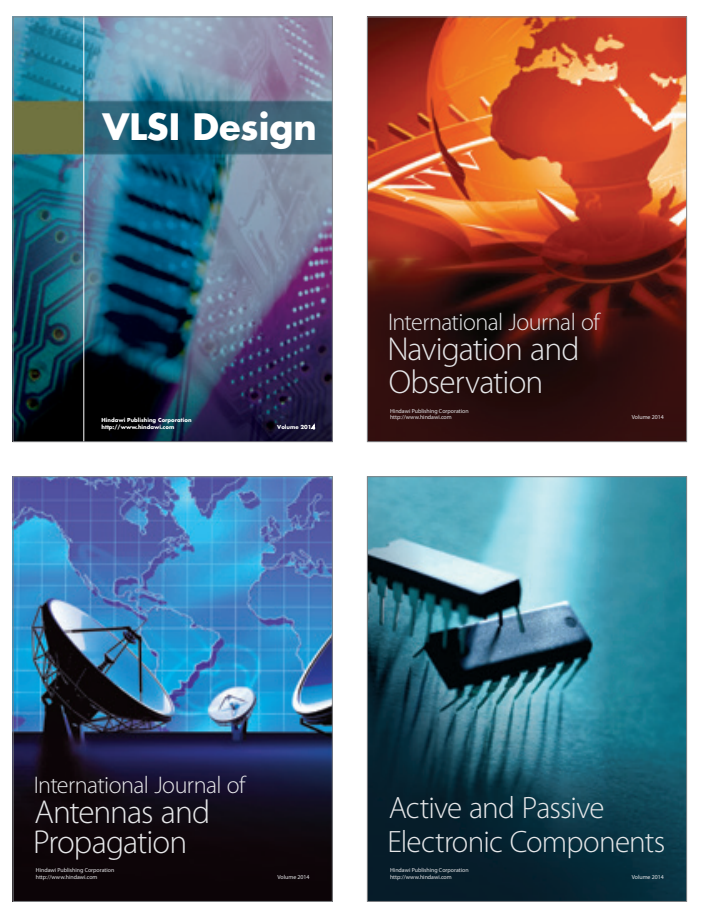
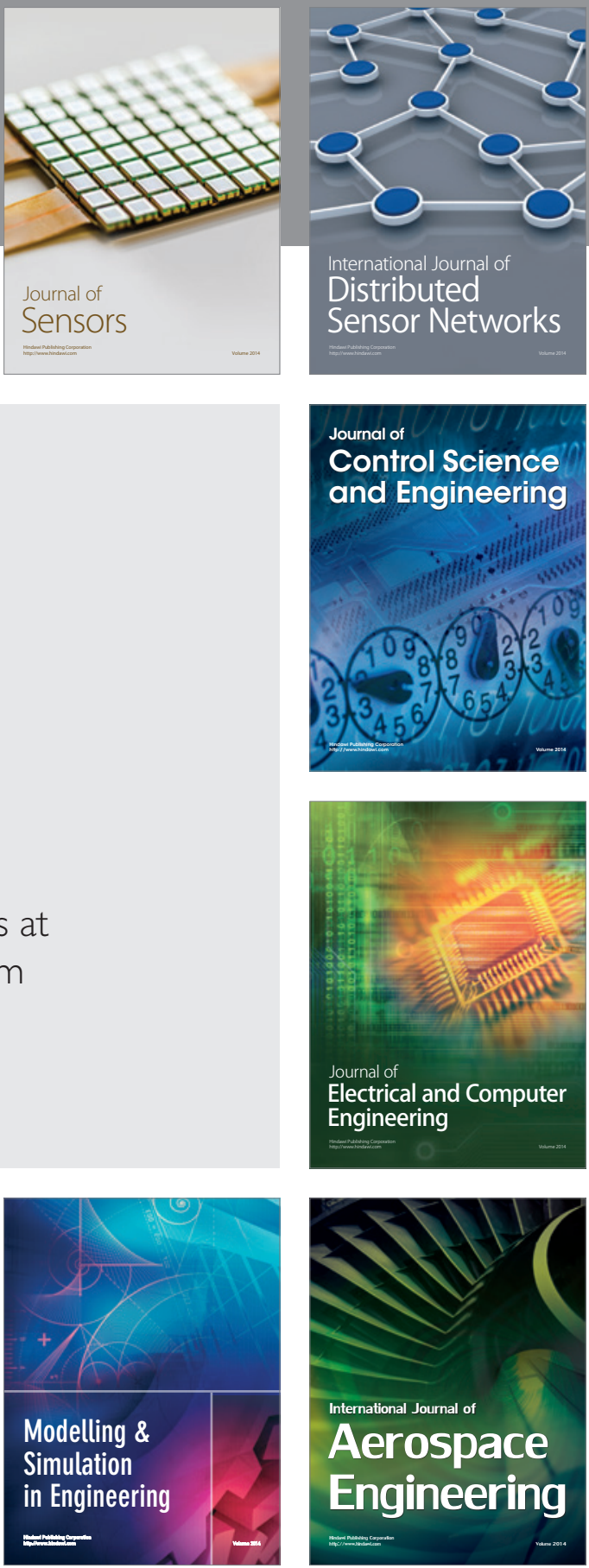

Journal of

Control Science

and Engineering
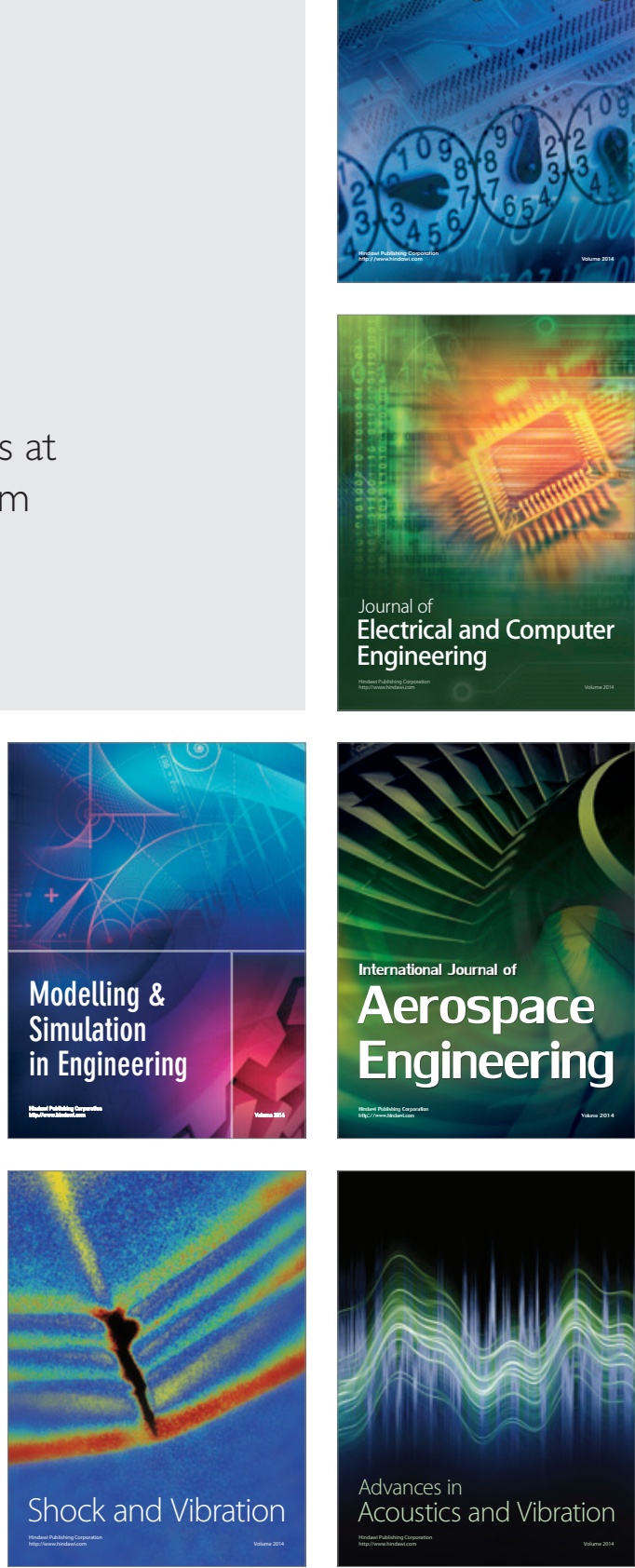